\title{
Decay of heavy-light hybrids in HQET sum rules
}

\author{
Tao Huang ${ }^{1,2 *}$, Hongying $\mathrm{Jin}^{2 \dagger}$ and Ailin Zhang ${ }^{2 \ddagger}$ \\ ${ }^{1}$ CCAST (World Laboratory), P. O. Box 8730, Beijing, 100080 \\ 2 Institute of High Energy Physics, P. O. Box 918, Beijing, 100039, P. R.China
}

\begin{abstract}
The decay widths of the $0^{++}$and $1^{-+}$heavy-light hybrids to $\mathrm{B}(\mathrm{D})$ and pion are calculated by using the QCD sum rules. The interpolated current of the hybrid is chosen as $g \bar{q} \gamma_{\alpha} G_{\alpha \mu}^{a} T^{a} h_{v}(x)$. In order to simplify the calculation and avoid the ambiguity of three-point correlation function, a two-point correlation function between the pion and vacuum is used instead. The decay width of the $0^{++} \rightarrow B(D)$ is about $12(16)$ $\mathrm{MeV}$ while the $1^{-+} \rightarrow B(D)$ is around $0.4(1.8) \mathrm{MeV}$. We keep the leading order of $1 / M_{Q}$ expansion in our calculation for convenience.
\end{abstract}

\section{Introduction}

It has been a long time for us to search the exotic hadrons such as the glueballs and hybrids. No candidate that has been found confirmedly so far. Recently, there are some evidences for the existence of hybrids resonance however. The E852 collaboration at BNL[1] has reported a $J^{p c}=1^{-+}$isovector resonance $\hat{\rho}(1405)$ in the reaction $\pi^{-} p \rightarrow \eta \pi^{0} n$ with the mass $1370 \pm 16_{-30}^{+50} \mathrm{MeV}$ and width $385 \pm 40_{-105}^{+65} \mathrm{MeV}$. Since the normal $\bar{q} q$ meson only has even spin in the channel $\left(\eta \pi^{0}\right)$, the state $1^{-+}$must be beyond the quark model. The Crystal Barrel collaboration has also claimed to find an evidence in $p \bar{p}$ annihilation which may be an resonance with a mass of $1400 \pm 20 \pm 20 \mathrm{MeV}$ and a width of $310 \pm 50_{-30}^{+50} \mathrm{MeV}$ [1]. Recently, the E852 collaboration put forth evidence for another $J^{p c}=1^{-+}$exotic meson $\hat{\rho}(1600)$ [2], decaying to $\rho \pi$, in the reaction $\pi^{-} p \rightarrow \pi^{+} \pi^{-} \pi^{-} p$, with a mass and width of $1593 \pm 8 \mathrm{MeV}$ and $168 \pm 20 \mathrm{MeV}$ respectively. If these experiments have been confirmed in the future, they will provide a very strong evidence for the existence of the constituent gluon.

Theoretically, the hybrids have been studied widely by various methods such as bag model[3], flux-tube model[4], QCD sum rules [5], lattice [6] and some other models [7]. However, there are few works about the heavy-light hybrids except for QCD sum rules 5 in which the spectrum of the heavy-light hybrid was given. Compared with $\bar{b} b$ and $\bar{c} c$ hybrid, the heavy-light hybrid is easier to be dealt with since the heavy quark effective theory (HQET) may work in such a system. As we know, HQET has led to much progress in the

*huangt@hptc5.ihep.ac.cn

†jhy@hptc5.ihep.ac.cn

${ }^{\ddagger}$ zhangal@hptc5.ihep.ac.cn 
theoretical understanding of the properties of hadrons 8 . In such a framework, many phenomenology methods become easier to be controlled. For instance, QCD sum rule in HQET has been applied to various calculations of physical parameters, such as the decay constants, form factors and decay widths 9 .

In the heavy-light hybrids case, the calculation in full QCD theory in Ref. [10] shows that the constituent of gluon gives a contribution more than $1.0 \mathrm{GeV}$ to the mass, so the "light freedom" is too heavy and the availability of $1 / M_{Q}$ expansion seems problematic. Let's consider this problem. In the framework of $1 / M_{Q}$ expansion, the mass of heavy hadron can be expressed as

$$
M=M_{Q}+m_{\text {light-freedom }}+\lambda^{2} / M_{Q}+\ldots,
$$

where the $\lambda$ have the dimension of mass and can be estimated by the mass split of the doublet such as $\left(B, B^{*}\right),\left(D, D^{*}\right)$ because of the heavy quark spin symmetry. For instance, $M_{B^{*}}-M_{B}=0.05 \mathrm{GeV}$, then $\lambda \approx 0.5 \mathrm{GeV}$, which is roughly equal to $m_{\text {light-freedom }}=$ $M_{B}-M_{b}=0.5 \mathrm{GeV}$ if we choose $M_{b}=4.7 \mathrm{GeV}$, therefore, the next-leading correction is around $\lambda / M_{b} \approx 1 / 10$. Similarly, one can obtain $\lambda \approx m_{\text {light-freedom }}=M_{D}-M_{c}=0.5 \mathrm{GeV}$ if one chooses $M_{c}=1.3 \mathrm{GeV}$, and $\lambda / M_{c} \approx 1 / 3$ for doublet $\left(D, D^{*}\right)$. From the calculation in Ref. [10], the mass split of $0^{++}$and $1^{-+}$is $0.5 \mathrm{GeV}$ for $b$ hybrid, so $\lambda=1.6 \mathrm{GeV} \sim$ $M_{b, 1^{-+}}-M_{b}$ and the next-leading correction is around $\lambda / m_{b} \approx 1 / 3$. For the $c$ hybrid, the mass split from Ref. [10 is $0.8 \mathrm{GeV}, \lambda=1.0 \mathrm{GeV}$, which deviates a little more from $m_{\text {light-freedom }}=M_{c, 1^{-+}}-m_{c} \approx 2.0 \mathrm{GeV}$ (it is also a little smaller than $1.6 \mathrm{GeV}$ ), and the next-leading correction $\lambda / M_{c} \sim 1.0$. Comparing with $\mathrm{B}$ and $\mathrm{D}$ mesons, we can think it is still safe to use $1 / M_{Q}$ expansion in $b$ hybrid system while it should be very careful to apply HQET to $c$ hybrids.

As to the decay property of hybrids, the results given by the QCD sum rules [11] gave a strong disfavor to the experiments. The decay width for some individual channel calculated by them is much lower than the reported experimental results. As we know, all the predictions in sum rules before is dependent on the three-point correlation function, so the uncontrolled ambiguity resulted from the double Borel transformation will be brought in and the infrared divergence in the soft pion limit will appear too. To avoid these disadvantages, we make use of the two-point correlation function between the pion and vacuum in our derivation instead of the normal three-point correlation function. Besides, it simplifies our calculation.

In this paper, we employ the HQET sum rules to calculate the decay width of the $0^{++}$ and $1^{-+}$hybrids to $B(D)$ meson and pion. For convenience, we keep only the leading order of $1 / M_{Q}$ expansion in our calculation, which may make some deviation in the $c$ hybrid case. Our numerical results show that the decay width of $0^{++} \rightarrow B(D)$ is about $12(16) \mathrm{MeV}$ while the decay width of $1^{-+} \rightarrow B(D)$ is only $0.4(1.8) \mathrm{MeV}$.

The paper is organized as follows. The analytic formalism of HQET sum rules for the decay of hybrid is given in Sec. 2. In Sec. 3, we calculate some pion's matrix element which is necessary in the sum rules for the calculation of decay width. The numerical results of the decay width were obtained in Sec. 4. We give the conclusion and discussion in the last section. 


\section{HQET sum rules for the decay of heavy-light hybrid mesons}

In this paper, we consider the following processes

$$
\begin{gathered}
H_{b}\left(0^{++}\right)(k) \longrightarrow B\left(0^{-+}\right)(k-q)+\pi^{ \pm}(q), \\
H_{b}\left(1^{-+}\right)(k) \longrightarrow B\left(0^{-+}\right)(k-q)+\pi^{ \pm}(q),
\end{gathered}
$$

where the $H_{b}\left(0^{++}\right)(k)$ represents the hybrid with $b$ quark and momentum $k$, which has $J^{p c}=0^{++}, H_{b}\left(1^{-+}\right)(k)$ represents the hybrid with $b$ quark and momentum $k$, which has $J^{p c}=1^{-+}$. Here, the electric charges of the mesons except for pion have not been written out explicitly. The cases of $H_{c}\left(0^{++}\right) \rightarrow D \pi^{ \pm}$and $H_{c}\left(1^{-+}\right) \rightarrow D \pi^{ \pm}$are completely analogous. To calculate the decay widths of these processes, we consider the following two-point correlator

$$
A_{\nu}\left(\omega^{\prime}, \omega, v\right)=i \int d x e^{i k x}\left\langle\pi^{ \pm}(q)\left|T\left\{j_{1 \nu}(x), j_{2}(0)\right\}\right| 0\right\rangle=A\left(\omega^{\prime}, \omega\right) v_{\nu}+B\left(\omega^{\prime}, \omega\right)\left(-q_{\nu}+q \cdot v v_{\nu}\right)
$$

Where $j_{1 \nu}(x)=g \bar{q} \gamma_{\mu} G_{\mu \nu}^{a} T^{a} h_{v}(x), j_{2}(x)=\bar{h}_{v} \gamma_{5} q(x), h_{v}$ is the heavy quark effective field with four velocity $v, A\left(\omega^{\prime}, \omega\right)$ and $B\left(\omega^{\prime}, \omega\right)$ are scalar functions of $\omega$ and $\omega^{\prime}$, where $\omega$ and $\omega^{\prime}$ are defined as

$$
\omega=2 k \cdot v \quad, \quad \omega^{\prime}=2(k-q) \cdot v .
$$

$A\left(\omega^{\prime}, \omega\right)$ and $B\left(\omega^{\prime}, \omega\right)$ are determined through the spectral density saturated by the mesons corresponding to the interpolated currents, respectively. Here we mention that the interpolated current for the hybrid with a fixed $J^{p c}$ is not unique, different interpolated current corresponds to different state 5 .

Before carrying out the operator product expansion, we can simplize the correlation function Eq. (4) firstly. Since the free heavy quark propagator in $x$ representation in HQET is $\int_{0}^{\infty} d \tau \delta(x-v \tau) \frac{1+\chi}{2}$ and the interaction of the heavy quark with the gluon field $A_{\mu}$ in the leading order of $1 / M_{Q}$ expansion is $g \bar{h} v \cdot A h$. Then in the fixed-point gauge $x_{\mu} A_{\mu}=0$ (which will be used throughout this paper), the full propagator of the heavy quark $\langle 0| T(h(x) \bar{h}(0)|0\rangle$ in the leading order of $1 / M_{Q}$ expansion is the same as the free one. Then the correlator (4) reduces to the one including only light quarks.

Performing the OPE in $A_{\nu}$, we get

$$
\begin{aligned}
A_{\nu} & =i \int d x e^{i k x}\left\langle\pi^{ \pm}(q)\left|T\left\{j_{1 \nu}(x), j_{2}(0)\right\}\right| 0\right\rangle \\
& =-\int d x e^{i(k-q) x} \int d \tau \delta(x-v \tau) \operatorname{Tr}\left\{\Gamma\left\langle\pi^{ \pm}(q)\left|G_{\mu \nu}(0) q(-x) \bar{q}(0)\right| 0\right\rangle\right\} \\
& =-\int d x e^{i \omega^{\prime} \tau / 2} \operatorname{Tr}\left\{\Gamma\left\{\left\langle\pi^{ \pm}(q)\left|G_{\mu \nu} q \bar{q}\right| 0\right\rangle-\left\langle\pi^{ \pm}(q)\left|\tau v \cdot D q \bar{q} G_{\mu \nu}\right| 0\right\rangle\right\}\right\}
\end{aligned}
$$

where $\Gamma=\gamma_{\mu} \frac{1+\psi}{2} \gamma_{5}$ and $G_{\mu \nu}=i g G_{\mu \nu}^{a} T^{a}$. In deriving the last equation in (6), the quark field $q(-x)$ is expanded around zero and only the first two terms are kept, the numerical results in section. 4 assure it a good expansion. We stress here that, since the perturbative theory does not break chiral symmetry $\partial_{\mu} j_{\mu}^{\pi}=0$, there is no perturbative contribution to the sum 
rules in (6) in the chiral limit. The two matrix elements in (6) will be obtained through another suitable sum rules with the method used in Ref. [12]. Their detailed expressions will be given in the next section.

On the other side, $A\left(\omega^{\prime}, \omega\right)$ and $B\left(\omega^{\prime}, \omega\right)$ can be represented with the spectral density through the dispersion relations. Because the perturbative contribution vanish, we suppose that the contribution of continuum states vanishes too.

From the definition of Eq. (5), we have

$$
\omega-\omega^{\prime}=2 q \cdot v
$$

and one has to make the double Borel transformation on the two variables $\omega$ and $\omega^{\prime}$. The soft pion approximation was used in the spectral density in Ref. [9], which deduced functions of single variable. However, in the case of heavy-light hybrid's decay, the soft pion approximation is not good since the pion momentum may be large. Here we make a more reasonable approximation

$$
\omega-\omega^{\prime}=2 q \cdot v \approx 2\left(\Lambda-\Lambda^{\prime}\right)
$$

in the infinite heavy quark mass limit, where $\Lambda \sim m_{H}-M_{Q}$ and $\Lambda^{\prime} \sim m_{\text {meson }}-M_{Q}$. Eq. (ㅍ) can be obtained from

$$
k^{2}+q^{2}-(k-q)^{2}=2 k \cdot q \approx 2 M_{Q} q \cdot v
$$

as $M_{Q} \rightarrow \infty$. We find that the difference between $\omega$ and $\omega^{\prime}$ is around $1-2 \mathrm{GeV}$ in the case of heavy-light hybrid. With the single pole terms and double pole term included in the spectral density, then $A\left(\omega^{\prime}, \omega\right)$ and $B\left(\omega^{\prime}, \omega\right)$ are expressed as functions of the single variable $\omega^{\prime}$, respectively

$$
\begin{aligned}
& A\left(\omega^{\prime}\right)=\frac{f_{H^{+}} f_{m} g_{H^{+} m \pi} m_{H^{+}}^{4} m_{m}^{2}}{\left(2 \Lambda^{\prime}-\omega^{\prime}\right)^{2} M_{Q}^{3}}+\frac{c_{0}}{2 \Lambda^{\prime}-\omega^{\prime}}, \\
& B\left(\omega^{\prime}\right)=\frac{f_{H^{-}} f_{m} g_{H^{-} m \pi} m_{H^{-}}^{4} m_{m}^{2}}{\left(2 \Lambda^{\prime}-\omega^{\prime}\right)^{2} M_{Q}^{3}}+\frac{c_{1}}{2 \Lambda^{\prime}-\omega^{\prime}},
\end{aligned}
$$

where $m_{H}, m_{m}$ and $M_{Q}$ are the mass of the hybrid, meson, and heavy quark, respectively. $c_{0}$ and $c_{1}$ in the above equations are constants. $f_{i}$ are decay constants and $g_{H^{ \pm} m \pi}$ refers to decay amplitudes. For convenience, they are defined in full QCD theory as below

$$
\begin{array}{rll}
\left\langle 0\left|j_{H}\right| H\left(0^{++}\right)\right\rangle=f_{H^{+}} m_{H^{+}}^{3} k_{\nu} & , \quad\left\langle 0\left|j_{H}\right| H\left(1^{-+}\right)\right\rangle=f_{H^{-}} m_{H^{-}}^{4} \epsilon_{\nu}, \\
\left\langle 0\left|j_{D}\right| D\right\rangle=-i f_{D} m_{D}^{2} / M_{c} & , \quad\left\langle 0\left|j_{B}\right| B\right\rangle=-i f_{B} m_{B}^{2} / M_{b}, \\
\left\langle\pi^{ \pm}(q) D \mid H\left(0^{++}\right)\right\rangle=g_{H^{+} D \pi} & ,\left\langle\pi^{ \pm}(q) D \mid H\left(1^{-+}\right)\right\rangle=g_{H^{-} D \pi} \epsilon \cdot q, \\
\left\langle\pi^{ \pm}(q) B \mid H\left(0^{++}\right)\right\rangle=g_{H^{+} B \pi} & , \quad\left\langle\pi^{ \pm}(q) B \mid H\left(1^{-+}\right)\right\rangle=g_{H^{-} B \pi} \epsilon \cdot q .
\end{array}
$$

Therefore, only one variable Borel transformation is needed. Taking the Borel transformation

$$
\begin{gathered}
\frac{1}{\tau} \hat{B}_{\tau}^{\omega^{\prime}}=\lim _{\substack{n \rightarrow \infty \\
-\omega^{\prime} \rightarrow \infty}} \frac{\omega^{\prime n}}{\Gamma(n)}\left(-\frac{d}{d \omega^{\prime}}\right)^{n} \\
\tau=-\omega^{\prime} / \text { fixed }
\end{gathered}
$$


on both sides of the sum rules and differentiate the results in the variable $1 / \tau$, we get the expression for the decay amplitudes

$$
\begin{aligned}
g_{H^{+} m \pi} & =\frac{M_{Q}^{3}}{f_{H^{+}} f_{m} m_{H^{+}}^{4} m_{m}^{2}}\left[2 \Lambda^{\prime} A^{\prime}(\tau)+A_{0}\right] e^{2 \Lambda^{\prime} / \tau}, \\
g_{H^{-} m \pi} & =\frac{M_{Q}^{3}}{f_{H^{-}} f_{m} m_{H^{-}}^{4} m_{m}^{2}}\left[2 \Lambda^{\prime} B^{\prime}(\tau)+B_{0}\right] e^{2 \Lambda^{\prime} / \tau},
\end{aligned}
$$

where the $A^{\prime}(\tau)$ and $B^{\prime}(\tau)$ are the Borel transformed function of $A\left(\omega^{\prime}\right)$ and $B\left(\omega^{\prime}\right)$, respectively. Their expressions are obtained

$$
\begin{aligned}
& A^{\prime}(\tau)=4 \sqrt{2}\left\{\left[3 b_{1}-2\left(m_{H}-m_{m}\right)^{2} d_{2}\right]+\left[\left(m_{H}-m_{m}\right) F_{1} / 4+F_{3} / 16\right] \frac{1}{\tau}\right\} \\
& B^{\prime}(\tau)=4 \sqrt{2}\left[2\left(m_{H}-m_{m}\right) d_{2}+F_{1} /(12 \tau)\right],
\end{aligned}
$$

and

$$
\begin{aligned}
A_{0} & =4 \sqrt{2}\left[\left(m_{H}-m_{m}\right) F_{1} / 4+F_{3} / 16\right], \\
B_{0} & =\frac{\sqrt{2} F_{1}}{3}
\end{aligned}
$$

where the parameters $b_{i}, d_{i}$ and $F_{i}$ will be given in the next section.

Once the decay amplitudes are determined, it's straightforward to obtain the decay widths of the processes (2) and (3).

\section{The sum rules for pion's matrix elements}

The key to the sum rules is the determination of the two pion's matrix elements in (6). The first pion's matrix element $\left\langle\pi^{ \pm}(q)\left|G_{\mu \nu} q \bar{q}\right| 0\right\rangle$ has already been given in Ref. [9] (d) as

$$
\begin{aligned}
\left\langle\pi^{i}(q)\left|D_{\mu} D_{\nu} q_{\alpha}^{a}(0) \bar{q}_{\beta}^{b}(0)\right| 0\right\rangle= & i\left\{\left[g_{\mu \nu}\left(a_{1}+a_{2} \phi\right)-i \sigma_{\mu \nu} b_{1}+i \varepsilon_{\mu \nu \rho \sigma} \gamma^{\rho} q^{\sigma} \gamma_{5} b_{2}\right.\right. \\
& +\left(q_{\mu} \gamma_{\nu}+q_{\nu} \gamma_{\mu}\right) c_{1}+\left(q_{\mu} \gamma_{\nu}-q_{\nu} \gamma_{\mu}\right) d_{1} \\
& +i\left(q_{\mu} \sigma_{\lambda \nu}+q_{\nu} \sigma_{\lambda \mu}\right) q^{\lambda} c_{2}+i\left(q_{\mu} \sigma_{\lambda \nu}-q_{\nu} \sigma_{\lambda \mu}\right) q^{\lambda} d_{2} \\
& \left.\left.+\left(e_{1}+e_{2} \not\right) q_{\mu} q_{\nu}\right] \gamma_{5}\right\}_{\alpha \beta}\left(\frac{\tau_{i}}{2}\right)_{a b}
\end{aligned}
$$

where the definition and the numerical results of the coefficients can be found in the same Ref.. From the Lorentz covariance, the matrix element $\left\langle\pi(q)^{ \pm}\left|\left(D_{\alpha} q\right)_{j}^{i} \bar{q} G_{\beta \gamma}\right| 0\right\rangle$ can be written as

$$
\begin{aligned}
\left\langle\pi^{ \pm}(q)\left|D_{\alpha} q \bar{q} G_{\beta \gamma}\right| 0\right\rangle= & \left\{i f_{1} q_{\alpha} \sigma_{\beta \gamma}-f_{2} \gamma_{\alpha}\left[q_{\beta} \gamma_{\gamma}-q_{\gamma} \gamma_{\beta}\right]+i f_{3} \gamma_{\alpha} \sigma_{\beta \gamma}-f_{4}\left(g_{\alpha \beta} q_{\gamma}-g_{\alpha \gamma} q_{\beta}\right)\right. \\
& \left.-f_{5}\left(g_{\alpha \beta} \gamma_{\gamma}-g_{\alpha \gamma} \gamma_{\beta}\right)\right\} \gamma_{5}+i f_{6} \epsilon_{\alpha \beta \gamma \rho} q_{\rho}-f_{7} q_{\alpha}\left(q_{\beta} \gamma_{\gamma}-q_{\gamma} \gamma_{\beta}\right)
\end{aligned}
$$

where $f_{i}$ are some constants to be determined. By using the motion equation in the chiral limit and performing some special traces, we can obtain the following equations about $f_{i}$

$$
\begin{aligned}
f_{1}=-\frac{1}{4} F_{1}, \quad f_{2}=\frac{1}{12}\left(F_{1}-F_{2}\right), \quad f_{3}=\frac{1}{48} F_{3}, \\
f_{4}=\frac{1}{12} F_{1}-\frac{1}{3} F_{2}, \quad f_{5}=-\frac{1}{24} F_{3}, \quad f_{6}=\frac{1}{4} F_{1},
\end{aligned}
$$


and $f_{7}$ vanishes. Where $F_{1}, F_{2}$ and $F_{3}$ are some constants defined as

$$
\begin{aligned}
\left\langle\pi^{ \pm}(q)\left|\bar{q} G_{\mu \nu} D_{\alpha} q\right| 0\right\rangle & =g \varepsilon_{\mu \nu \alpha \rho} q_{\rho} F_{1}, \\
\left\langle\pi^{ \pm}(q)\left|\bar{q} \gamma_{5} G_{\mu \nu} D_{\alpha} q\right| 0\right\rangle & =i g\left(g_{\alpha \nu} q_{\mu}-g_{\alpha \mu} q_{\nu}\right) F_{2}, \\
\left\langle\pi^{ \pm}(q)\left|\bar{q} \gamma_{5} \gamma_{\mu} G_{\mu \nu} D_{\nu} q\right| 0\right\rangle & =i g F_{3} .
\end{aligned}
$$

In order to get the numerical results of the constants $F_{i}$, we take advantage of some other suitable sum rules, through which the constants $F_{i}$ can be obtained by the following correlation functions, respectively

$$
\begin{aligned}
\Pi_{\mu \nu \alpha}^{1}(q) & =i \int d x e^{i q \cdot x}\left\langle 0\left|T\left\{\bar{q} G_{\mu \nu}^{a} T^{a} D_{\alpha} q(x), \bar{q} \gamma_{5} q(0)\right\}\right| 0\right\rangle, \\
\Pi_{\mu \nu \alpha}^{2}(q) & =i \int d x e^{i q \cdot x}\left\langle 0\left|T\left\{\bar{q} \gamma_{5} G_{\mu \nu}^{a} T^{a} D_{\alpha} q(x), \bar{q} \gamma_{5} q(0)\right\}\right| 0\right\rangle, \\
\Pi_{\rho}^{3}(q) & =i \int d x e^{i q \cdot x}\left\langle 0\left|T\left\{\bar{q} \gamma_{5} \gamma_{\mu} G_{\mu \nu}^{a} T^{a} D_{\nu} q(x), \bar{q} \gamma_{\rho} \gamma_{5} q(0)\right\}\right| 0\right\rangle .
\end{aligned}
$$

where the $\bar{q}$ or $q$ refer to the $\mathrm{u}$, d quark or antiquark corresponding to which in the $\pi^{ \pm}$.

Keeping the matrix elements of OPE up to dimension six and omiting the radiative correction, we obtain the Borel transformed functions

$$
\begin{aligned}
& F_{1}(M)=\left\{-\frac{2 \alpha_{s}}{9(4 \pi)^{3}}\left(M^{2}\right)^{2}+\frac{1}{24}\left\langle 0\left|\frac{\alpha_{s}}{\pi} G^{2}\right| 0\right\rangle+\frac{4 \pi}{27 M^{2}} \alpha_{s}\langle 0|\bar{q} q| 0\rangle^{2}\right\} \frac{\left(m_{u}+m_{d}\right) M^{2}}{f_{\pi} m_{\pi}^{2}} e^{m_{\pi}^{2} / M^{2}}(23) \\
& F_{2}(M)=-F_{1}(M), \\
& F_{3}(M)=\left\{\frac{-31 \alpha_{s}}{15(4 \pi)^{3}}\left(M^{2}\right)^{2}+\frac{1}{4}\left\langle 0\left|\frac{\alpha_{s}}{\pi} G^{2}\right| 0\right\rangle-\frac{4 \pi}{9 M^{2}} \alpha_{s}\langle 0|\bar{q} q| 0\rangle^{2}\right\} \frac{M^{2}}{f_{\pi}} e^{m_{\pi}^{2} / M^{2}} .
\end{aligned}
$$

where $M$ is the Borel transform parameter of the sum rules.

To obtain the numerical results, the constants and condensates are chosen as

$$
\begin{aligned}
f_{\pi}=132 \mathrm{MeV}, & m_{\pi}=140 \mathrm{MeV}, & m_{u}+m_{d}=15 \mathrm{MeV}, \\
\left\langle 0\left|\frac{\alpha_{s}}{\pi} G^{2}\right| 0\right\rangle=0.012 \mathrm{GeV}^{4}, \quad\langle 0|\bar{q} q| 0\rangle & =-(0.24 \mathrm{GeV})^{3}, & \alpha_{s}\langle 0|\bar{q} q| 0\rangle^{2}=8 \cdot 10^{-5} \mathrm{GeV}^{6}
\end{aligned}
$$

and where the $\alpha_{s}=0.4$.

The numerical results are showed as Fig. 1 and Fig. 2, where one can find stable platforms in the region $M \sim 1.1-1.6 \mathrm{GeV}$. they read

$$
F_{1}=-F_{2}=4.0 \cdot 10^{-3} \mathrm{GeV}^{4} \quad, \quad F_{3}=2.3 \cdot 10^{-2} \mathrm{GeV}^{5} .
$$

\section{4 the numerical results of the decay width}

Before going on the numerical calculation of the decay width, there are still some parameters necessary to be fixed. The heavy quark and meson masses are given as those in Ref. [13] and the decay constants of B and D mesons are chosen as Ref. [14]. The masses of the heavy-light hybrids have been computed in Ref. [10] in the full QCD theory though the b

quark mass $(4.23 \mathrm{GeV})$ and some other parameters were chosen unsuitablely, we just recite the results of their calculation in this paper. 
Table 1: Parameters input and the decay amplitudes.

\begin{tabular}{|c|c|c|c|c|c|}
\hline & $M_{Q}$ & $m_{m}$ & $f_{m}$ & $m_{H}\left(0^{++}\right)$ & $m_{H}\left(1^{-+}\right)$ \\
\hline b quark & $4.7 \mathrm{GeV}$ & $5.28 \mathrm{GeV}$ & $180 \mathrm{MeV}$ & $6.8 \mathrm{GeV}$ & $6.3 \mathrm{GeV}$ \\
\hline c quark & $1.3 \mathrm{GeV}$ & $1.87 \mathrm{GeV}$ & $190 \mathrm{MeV}$ & $4.0 \mathrm{GeV}$ & $3.2 \mathrm{GeV}$ \\
\hline & $f_{H\left(0^{++}\right)}$ & $f_{H\left(1^{-+}\right)}$ & $g_{H^{+} m \pi}$ & $g_{H^{-} m \pi}$ & \\
\hline b quark & 0.0012 & 0.0012 & $3.2 \mathrm{GeV}$ & 1.2 & \\
\hline c quark & 0.004 & 0.004 & $2.0 \mathrm{GeV}$ & 1.1 & \\
\hline
\end{tabular}

The decay constants of hybrids hadn't been determined in Ref. [10] yet, so we fix them through the parameters and formulae given in it as

$$
f_{i}^{2}=\frac{M^{2}}{m_{R}^{8}} e^{\frac{m_{R}^{2}}{M^{2}}} \prod_{i}\left(M^{2}\right),
$$

where $\Pi_{i}\left(M^{2}\right)$ refer to the functions corresponding to the $J^{p c}=0^{++}$and $J^{p c}=1^{-+}$states

$$
\begin{aligned}
\Pi_{s}\left(M^{2}\right) & =\frac{3 \alpha_{s}}{4 \pi^{3}} M^{6} \int_{0}^{1} d x \int_{0}^{1} d y x(1-x) y^{3}(3 y-2) e^{-\frac{m^{2}}{M^{2} x y}} \\
& -\frac{1}{8}\left\langle\frac{\alpha_{s}}{\pi} G^{2}\right\rangle M^{2} \int_{0}^{1} d x x^{2} e^{-\frac{m^{2}}{M^{2} x}}-\frac{\alpha_{s}}{\pi}\langle m \bar{q} q\rangle M^{2} \int_{0}^{1} d x x(1-x) e^{-\frac{m^{2}}{M^{2} x}}, \\
\Pi_{v}\left(M^{2}\right) & =\frac{3 \alpha_{s}}{4 \pi^{3}} M^{6} \int_{0}^{1} d x \int_{0}^{1} d y x(1-x) y^{4} e^{-\frac{m^{2}}{M^{2} x y}} \\
& +\frac{1}{24}\left\langle\frac{\alpha_{s}}{\pi} G^{2}\right\rangle M^{2} \int_{0}^{1} d x x(2-x) e^{-\frac{m^{2}}{M^{2} x}}-\frac{\alpha_{s}}{3 \pi}\langle m \bar{q} q\rangle M^{2} \int_{0}^{1} d x x(1-x) e^{-\frac{m^{2}}{M^{2} x}} .
\end{aligned}
$$

where $m_{R}$ is the mass of the hybrids, the Borel parameter $M$ is chosen in the region where both the masses and the decay constants have platform and the scale of the $\alpha_{s}$ is set at the Borel variable. Their results are given in the table 1 too.

With the help of Eq. (12) and Eq. (13), the numerical results of the decay amplitudes are shown as Fig. 3 and Fig. 4. The platform is not good, we choose the value around the region $\tau \sim 2.5 \mathrm{GeV}$, which is the suitable region in sum rules analysis, as our results. The numerical result shows that the next-leading contribution is less than $1 / 5$ of the leading term at this region, so the OPE is well performed. All the parameters and some results calculated are collected in table. 1.

As to the processes (2) and (3) we considered, with the help of the formulae for two body decay, the decay widths in the leading order of $1 / M_{Q}$ expansion are given as

$$
\Gamma\left(H\left(0^{++}\right) \rightarrow m\left(0^{-+}\right)+\pi\right)=\frac{g_{H m \pi}^{2}}{8 \pi} \frac{|q|}{m_{H}^{2}}=\frac{g_{H m \pi}^{2}}{16 \pi} \frac{m_{H}^{2}-m_{m}^{2}}{m_{H}^{3}},
$$




$$
\Gamma\left(H\left(1^{-+}\right) \rightarrow m\left(0^{-+}\right)+\pi\right)=\frac{g_{H m \pi}^{2}}{24 \pi} \frac{|q|^{3}}{m_{H}^{2}}=\frac{\left(m_{H}^{2}-m_{m}^{2}\right)^{3} g_{H m \pi}^{2}}{192 \pi m_{H}^{5}}
$$

When all the numerical results are put in, the decay width corresponding to processes (2) and (3) are obtained

$$
\begin{array}{cl}
\Gamma\left(H\left(0^{++}\right) \rightarrow B\left(0^{-+}\right)+\pi\right)=12 M e V, & \Gamma\left(H\left(0^{++}\right) \rightarrow D\left(0^{-+}\right)+\pi\right)=16 M e V \\
\Gamma\left(H\left(1^{-+}\right) \rightarrow B\left(0^{-+}\right)+\pi\right)=0.4 M e V, & \Gamma\left(H\left(1^{-+}\right) \rightarrow D\left(0^{-+}\right)+\pi\right)=1.8 M e V .
\end{array}
$$

As we know, the decay of hybrids appears to follow the almost universal selection rule that gluonic excitations cannot transfer angular momentum to the final states as relative angular momentum, that is to say, the decay of hybrids to $S+P$ final pairs of mesons are preferred, while the decay of hybrids to two S-wave mesons are suppressed [15]. But the selection rule is not absolute, in the flux tube and constituent glue models, it can be broken by wave function and relativistic effects, and the bag model predict that it is also possible that the excited quark loses its angular momentum to orbital angular momentum [16]. Though the results obtained here are much larger than those calculated for the similar decay modes in the light hybrids case [11], which depended on the construction of three point correlation function, these results couldn't be excluded by theory.

The reason that the decay widths of the $1^{-+}$hybrids are much smaller than those of the $0^{++}$hybrids results from the additional factor $q_{\pi}^{2} / m_{H}^{2}$ in the width formulae(28) compared with that of the $0^{++}$'s, since in the decay channel $H_{b, c}\left(1^{-+}\right) \rightarrow B(D) \pi$, the final states are in a relative $\mathrm{P}$-wave.

\section{Conclusion and Discussion}

In this paper, we discuss the decay of the heavy-light hybrids $H\left(0^{++}\right)$and $H\left(1^{-+}\right)$to $B(D) \pi$ by QCD sum rule approach in the framework of HEQT. With the help of the spectrum of the heavy-light hybrids 10 , we argue, in the $b$ hybrid case, convergence of $1 / M_{Q}$ expansion is similar as that in the $D$ meson case, while it is not so good for the $c$ hybrid. The two-point correlation function instead of the normal three-point correlation function is employed for the derivation, and the heavy quark freedom is extracted out of the correlation functions in the $x$ representation, which leads to the estimate of some pion's matrix elements and facilitates the calculation. In the leading order of $1 / M_{Q}$ expansion, the process of the calculation becomes much easier.

To find the hybrids, one approach is to look for an excess of observed state over the number predicted by the quark model, the other is to search for quantum numbers which cannot be accommodated in the quark model, so the experiments place main efforts on the exotic mesons' searching and the current experiments concern mainly the light hybrids sector, however, it's necessary to extend the search to the heavy-light hybrids sector. The calculation shows that the decay width of $H\left(0^{++}\right) \rightarrow B(D) \pi^{+}$is around $12(16) \mathrm{MeV}$, while the decay width $H\left(1^{-+}\right) \rightarrow B(D) \pi^{+}$is only $0.4(1.8) \mathrm{MeV}$. If people think the total width of hybrid is around $200 \mathrm{MeV}$, the channel $B(D) \pi$ may not be dominant in hybrid's decay, for instance, the channels $B, D\left(B^{*}, D^{*}\right) \eta^{\prime}$ may be larger because of QCD anomaly. The calculation of these and other channels is beyond this paper. 
There are some uncertainties unavoidable in this paper, which result from such as pole approximation, $1 / M_{Q}$ expansion(we keep only the leading order term) and OPE(we keep only the first two terms of OPE in (6)), omitting the higher orders would give some uncertainties. Besides, the calculation is dependent on the numerical results of the masses and decay constants of the hybrids taken from the Ref. [10], which were determined by some unpopular parameters. To follow the calculation of Ref. [10] with the new values of the parameters is beyond the goal of this paper. Certainly, it's necessary to identify the spectrum and decay width of heavy-light hybrids through other models and other sum rules, for example, light cone sum rules. Only when most of the models give detailed research on hybrids, can we declaim that we have a good understanding about the hybrids.

\section{Acknowledgment}

This work is supported in part by the national natural science foundation of P. R. China.

\section{References}

[1] D. R. Thompson et al. (E852 Collab.), Phys. Rev. Lett. 79 (1997) 1630.

A. Abele et al. (Crystal Barrel Collab.), Phys. Lett. B423 (1998) 175.

[2] G. S. Adams et al. (E852 Collab.), Phys. Rev. Lett. 81 (1998) 5760.

[3] T. Barnes, F. E. Close and F.de Viron, Nucl. Phys. B224 (1983) 241.

M. Chanowitz and S. Sharpe, Nucl. Phys. B222 (1983) 211.

[4] N. Isgur, R. Kokoski and J. Paton, Phys. Rev. Lett. 54 (1985) 869.

F. E. Close and P. R. Page, Nucl. Phys. B443 (1995) 233.

F. E. Close and P. R. Page, Phys. Rev. D52 (1995) 1706.

A. Donnachie and P. R. Page, Phys. Rev. D58 (1998): 114012.

[5] I. I. Balitsky, D. I. Dyakonov and A. V. Yung, Phys. Lett. B112 (1982) 71.

J. I. Latorre, P. Pascual and S. Narison, Z. Phys. C34 (1987) 347.

J. Govaerts, L. J. Reinders, P. Francken, X. Gonze and J. Weyers, Nucl. Phys. B284 (1987) 674.

Tao Huang, Hongying Jin and Ailin Zhang, Eur. Phys. J. C8 (1999) 465.

[6] C. Michael et al., Nucl. Phys. B347 (1990) 854; Phys. Lett. B142 (1984) 291; Phys. Lett. B129 (1983) 351.

Liverpool Univ. Report LTH-286(1992); Proceedings of the Workshop on QCD : 20 Years later, Aachen, Germany, 1992.

[7] T. Barnes, F. E. Close and E. S. Swanson, Phys. Rev. D52 (1995) 5242.

Yu. S. Kalashnikova and Yu. B. Yufryakov, Phys. Lett. B359 (1995) 175.

[8] E. Eichten and B. Hill, Phys. Lett. B234 (1990) 511; B243 (1990) 427.

H. Georgi, Phys. Lett. B240 (1990) 447. 
N. Isgur and M. B. Wise, Phys. Lett. B232 (1989) 113; B237 (1990) 527.

A. F. Falk, H. Georgi, B. Grinstein and M. B. Wise, Nucl. Phys. B343 (1990) 1.

[9] (a). M. Neubert, Phys. Rev. D47 (1993) 4063.

(b). E. Bagan, P. Ball, V. M. Braun and H. G. Dosch, Phys. Lett. B278 (1992) 457.

(c). P. Colangelo, F. De Fazio, G. Nardulli, N. Di Bartolomeo and R. Gatto, Phys. Rev. D52(1995) 6422.

(d). Y. B. Dai, C. S. Huang, M. Q. Huang, H. Y. Jin and C. Liu, Phys. Rev. D58 (1998) 094032 .

[10] J. Govaerts, L. J. Reinders and J. Weyers, Nucl. Phys. B262 (1985) 575.

[11] F. de. Viron and J. Govaerts, Phys. Rev. Lett. 53 (1984) 2207.

J. I. Latorre, P. Pascual and S. Narison. Z. Phys. C34 (1987) 347.

[12] V. A. Novikov, M. A. Shifman, A. I. Vainshtein, M. B. Voloshin and V. I. Zakharov, Nucl. Phys. B237(1984) 525.

[13] C. Caso it al(Particle Data Group), Eur. Phys. J. C3 (1998) 1.

[14] R. Rückl, hep-ph/9810338.

[15] P. R. Page, Phys. Lett. B401 (1997) 313.

P. R. Page, Phys. Lett. B402 (1997) 183.

[16] S. Godfrey, J. Napolitano, hep-ph/9811410, submitted to Rev. Mod. Phys. 


\section{Figure caption}

Figure 1: $F_{1}$ versus Borel variable M.

Figure 2: $F_{3}$ versus Borel variable $\mathrm{M}$.

Figure 3: Decay amplitude of $0^{++}$heavy-light hybrids versus Borel variable $\tau$.

Figure 4: Decay amplitude of $1^{-+}$heavy-light hybrids versus Borel variable $\tau$. 


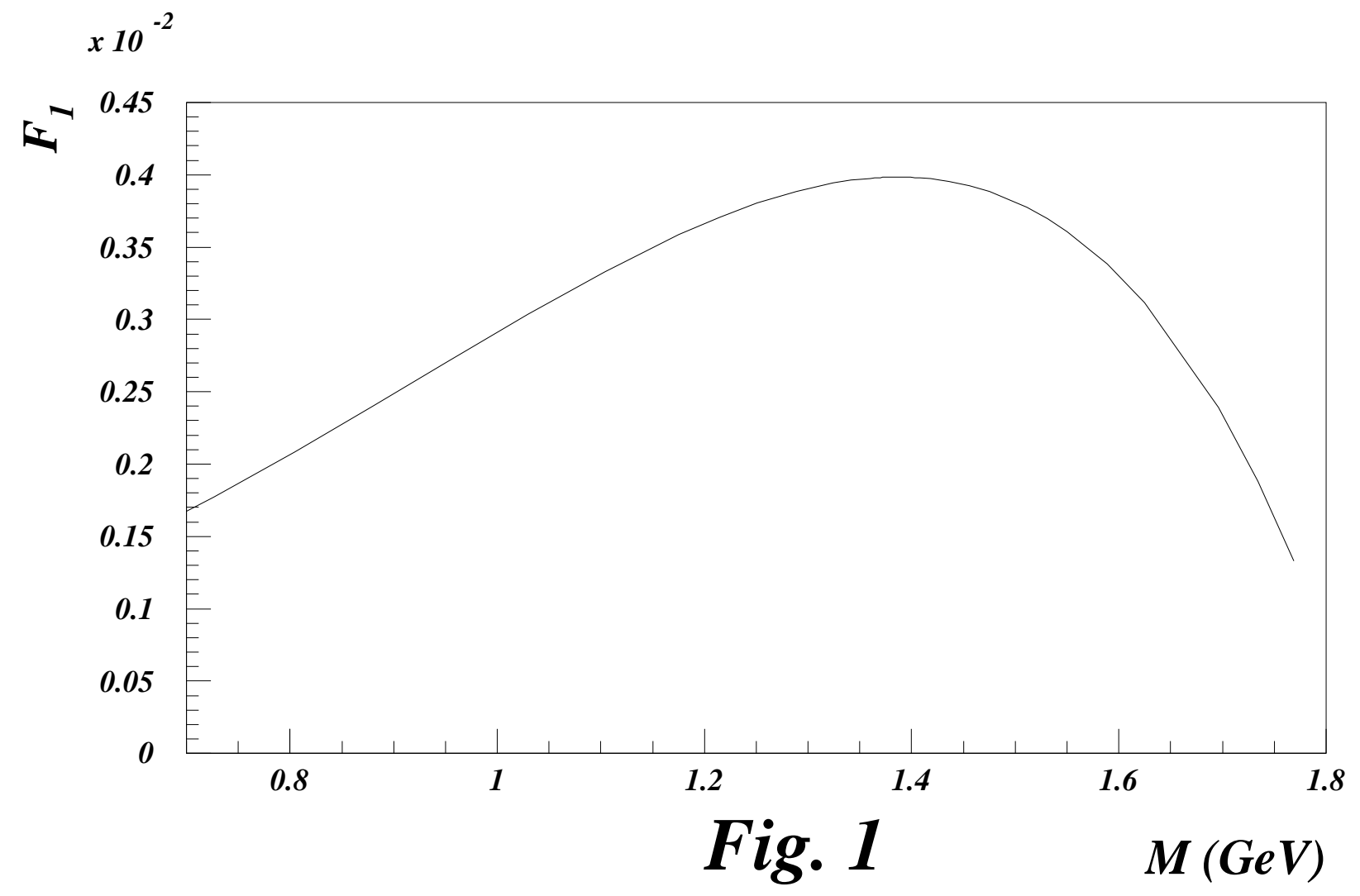




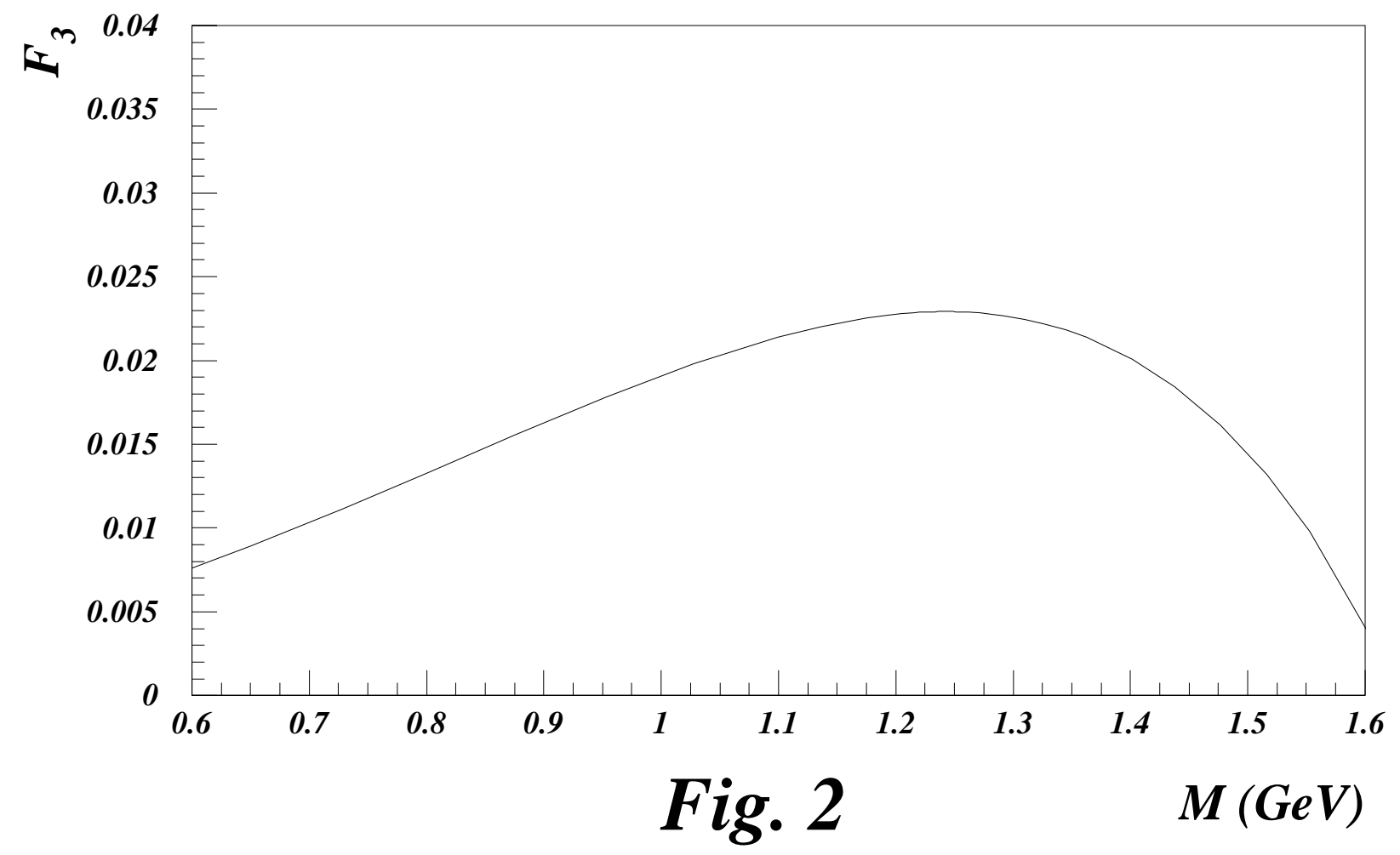




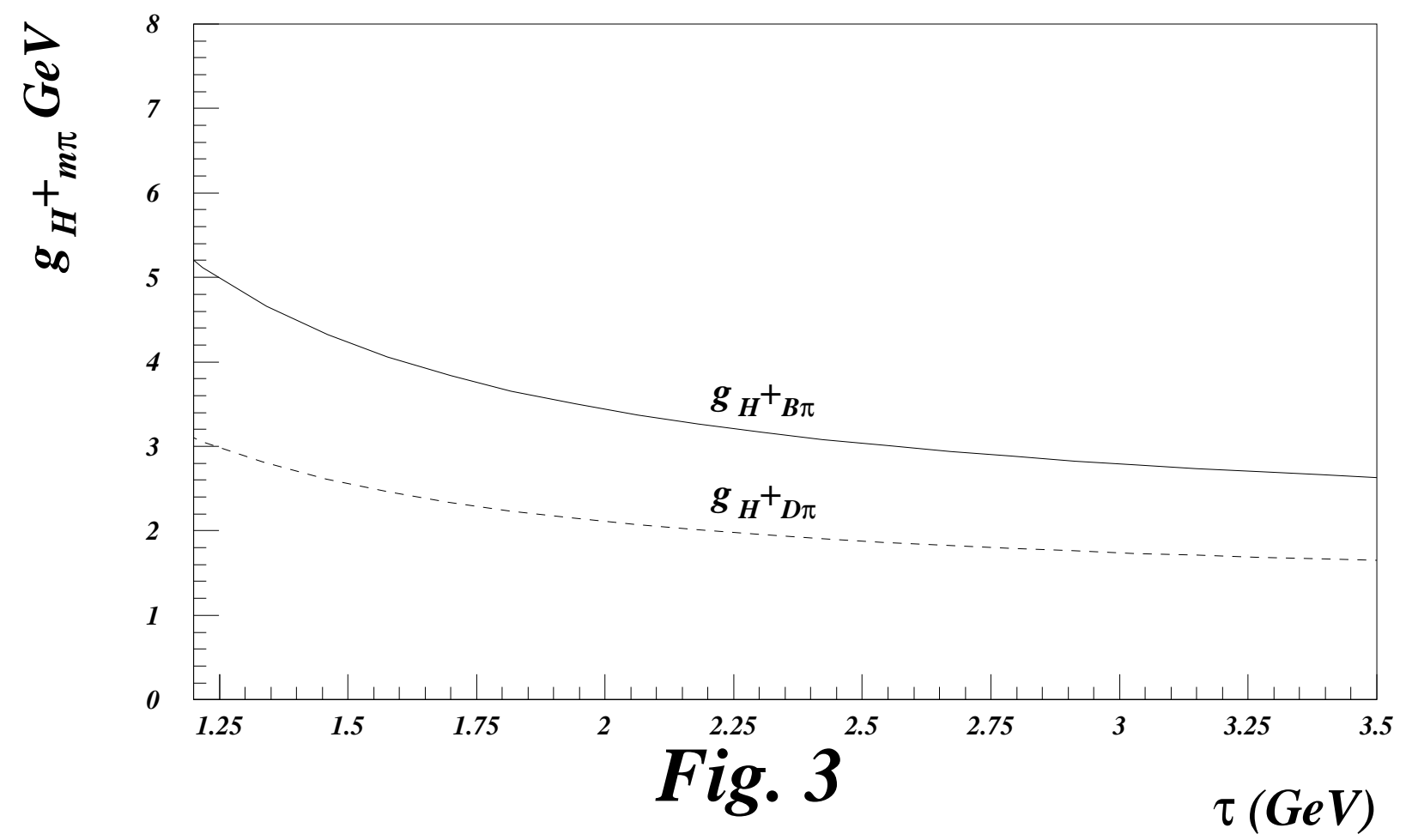




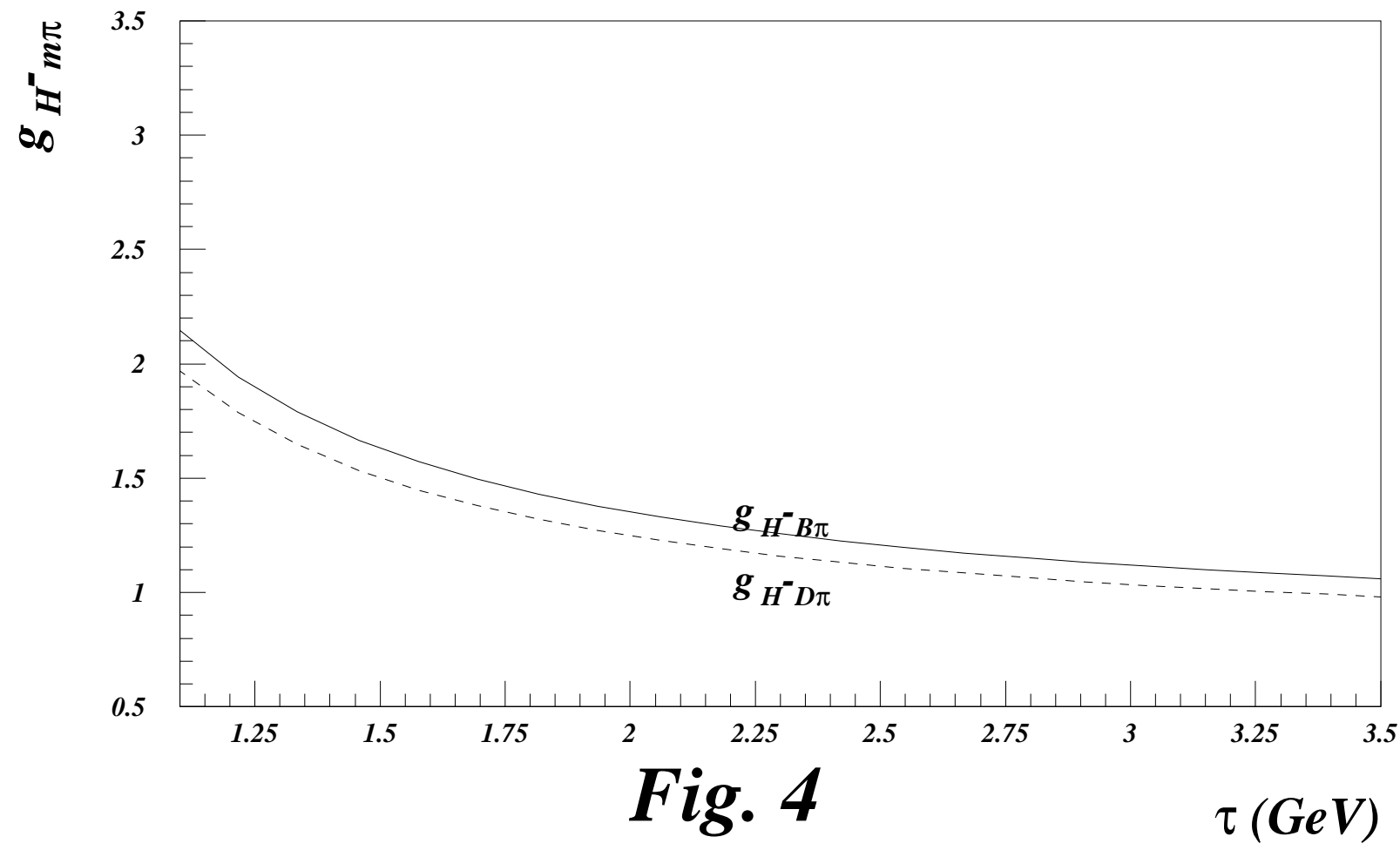

